\title{
A Study of undulator magnets characterization using the Vibrating Wire technique
}

\author{
Alexander. Temnykh ${ }^{\mathrm{a}}$, Yurii Levashov ${ }^{\mathrm{b}}$ and Zachary Wolf ${ }^{\mathrm{b}}$ \\ ${ }^{a}$ Cornell University, Laboratory for Elem-Particle Physics, Ithaca, NY 14850, USA \\ ${ }^{\mathrm{b}}$ SLAC National Accelerator Laboratory, Menlo Park, CA 94025, USA
}

\begin{abstract}
.
The vibrating wire $(\mathrm{VW})$ technique employs a stretched wire as a magnetic field sensor. Because of the wire's small diameter $(\sim 0.1 \mathrm{~mm}$ or smaller) and because the wire can be supported from outside the magnet, this technique is very appealing for field measurements in small gap/bore undulators with small good field regions and with limited access to the tested field. In addition, in the case of elliptical undulators in which Hall probe (HP) measurements can be affected by the planar Hall effect, VW technique can be used as an independent method to verify and supplement HP measurements.

In this article we studied the potential of the VW technique for measurement of magnetic field errors and for prediction of beam trajectories in undulator magnets using a $3.8 \mathrm{~m}$ long LCLS undulator as a test bench. Introducing calibrated magnetic field distortion at various locations, we measured the sensitivity and spatial resolution of the method. The method demonstrated $0.9 \mathrm{~mm}$ spatial resolution at a distance up to a few meters and $0.37 \mathrm{Gcm}$ sensitivity to the field integral. To compare Hall probe and Vibrating wire measurements side-by-side, we measured field errors in an LCLS undulator previously characterized by Hall probe measurements. The field errors found with the Vibrating Wire technique appeared to be in good agreement with errors measured with the Hall probe. Beam trajectory distortions calculated from both data sets are also in a good agreement.
\end{abstract}

\section{Introduction.}

The vibrating wire technique, developed in Ref [1], employs a stretched wire as a magnetic field sensing element. In the usual setup, see Fig.1, the wire is stretched through the region of the magnetic field to be tested and $\mathrm{AC}$ current with various frequencies is driven through the wire. The Lorentz forces generated by AC current flowing in the magnetic field cause the wire motion. The force distribution along the wire repeats the magnetic field profile. If the frequency of AC current is equal to the resonance frequency of one of the wire vibration modes, this mode (standing wave) will be excited with amplitude and phase (relative driving current) dependent on the field distribution. By measuring amplitudes and phases of various vibrating modes, one can reconstruct the field distribution along the wire. Detailed descriptions of the measurement procedure and field reconstruction algorithm can be found in Ref [1]. It should be noted that because of the resonance amplification of the wire motion and the use of the driving current as a reference for lock-in wire motion detection, the VW method is very sensitive to small magnetic fields.

The Vibrating Wire technique has been used on several occasions. In Laboratory for Elementary Particle Physics (Cornell University) VW technique was applied for magnetic center finding and alignment of final focusing quadrupole magnets of the Cornell Electron Storage Ring, Ref [2]; 
for accurate finding of solenoid magnetic axis Ref [3]; for permanent magnet wiggler end transitions tuning and magnetic field measurement along wiggling beam trajectory, Ref [4]. In Stanford Linear Accelerator Center National Laboratory, the method was used for finding the magnetic axis of LCLS quadrupoles Ref [5]. In National Synchrotron Radiation Research Center (Taiwan), VW set up was proposed to align Taiwan Photon Source quadropole magnets placed on girder, Ref [6]. Ref [7] describes the use of the VW technique for precise alignment of NSLS-II quadrupole and sextupole magnets at Brookhaven National Laboratory.

It should be noted that in the majority of the applications listed above, only a few harmonics of the wire vibration were used. The field reconstruction algorithm was not employed.

The present study was motivated by the need for the field error measurement in small gap/bore undulator magnets as well as by a request for independent, from Hall probe, techniques for field measurement in elliptical undulators where the planar Hall probe effect may significantly distort results. In this study we took all advantages of the field reconstruction.

\section{Setup.}

The setup is shown in Fig.1. A copper-beryllium wire was stretched through the $3.4 \mathrm{~m}$ long LCLS undulator [10]. The undulator had $6.8 \mathrm{~mm}$ gap, $3 \mathrm{~cm}$ period, hybrid type magnetic structure with $\sim 1.25 \mathrm{~T}$ peak field. The wire was extended by $838 \mathrm{~mm}$ and $381 \mathrm{~mm}$ beyond the magnet, and the ends were fixed on stands. Close to the left end of the wire, two LED-phototransistor assemblies (H21A1 from Newark Electronics) were employed as vertical and horizontal wire position sensors. The horizontal sensor was placed at $\mathrm{z}=152 \mathrm{~mm}$, vertical at $\mathrm{z}=177 \mathrm{~mm}$. The tension mechanism was located on the opposite side.
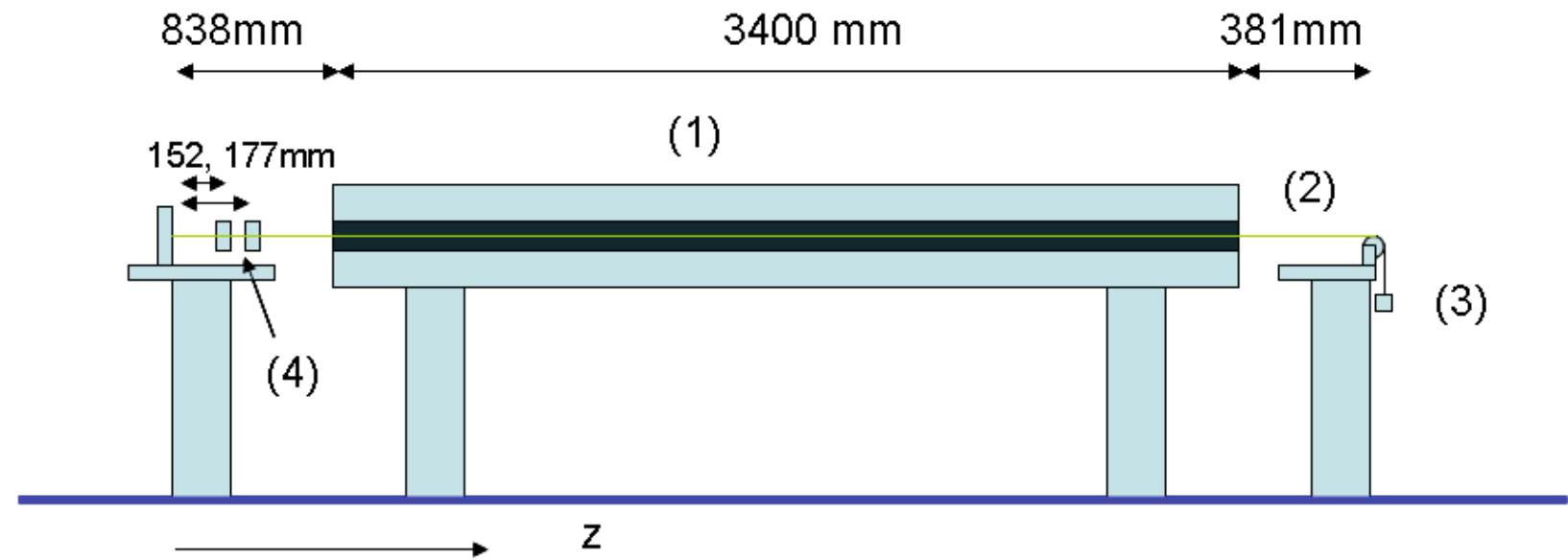

Figure 1:

VW setup. Here are (1) - LCLS undulator, (2) - 4617mm long 100 micron diameter copper-beryllium wire, (3) - tension mechanism, (4) - wire position optical sensors.

A wave form generator HP33120A controlled by IBM T42 laptop trough GPIB interface was used to drive $\mathrm{AC}$ current through the wire. In all measurements the current amplitude did not exceed 100mA. For the data acquisition we used a DAQcard-6024E from National Instruments. A program based on "LabView" controlled the wave form generator, recorded the signal, and made initial processing. Subsequently, the data was analyzed with MatLab 7.1. The first vibration mode resonance frequency of the wire was found to be around $30 \mathrm{~Hz}$. That indicated $0.34 \mathrm{~mm}$ sag at wire center, see expression (10) in Ref. [1]. 


\section{Experiments and results.}

The main goal for the experimental program was to explore the potential of the VW techniques using the LSLC undulator as a test bench. Knowing the potential, one can develop the practical application. The first experiment was the test of the measurement repeatability.

\section{Repeatability test.}

With LCLS undulator (S/N 1) on the bench, we measured the magnetic field using 25 harmonics of the wire vibration. Fig. 2 and 3 depict data of three independent measurements.

Measurements (2) and (3), were done subsequently within one hour; measurement (4) was done the next day. The measured amplitudes of the standing waves up to 25 orders are shown on Fig. 2. Positive values mean the waves are in phase with driving current, negative ones indicate 180 degrees phase. Plots in Fig. 3 present vertical and horizontal magnetic fields reconstructed from the wire vibration modes following the algorithm developed in Ref.[1]. It should be noted that in Vibrating Wire field measurements the tested field is automatically integrated over the shortest used half-wavelength. In the given measurements the shortest half-wavelength was $\sim 18 \mathrm{~cm}$ which is approximately equal to 6 undulator periods. This integration zeroed ideal field and left only the error field components.
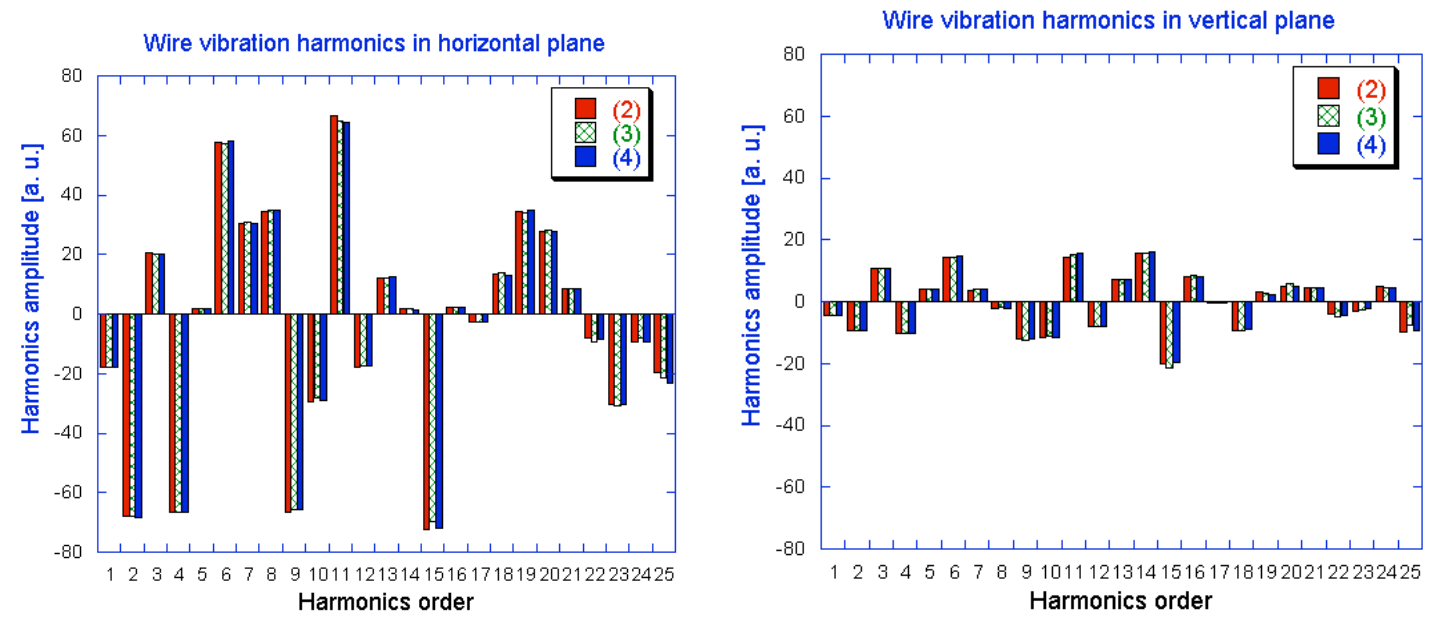

Figure 2:

Amplitudes of the wire vibrating modes (standing waves) with phase in horizontal (left) and in vertical (right) planes. 

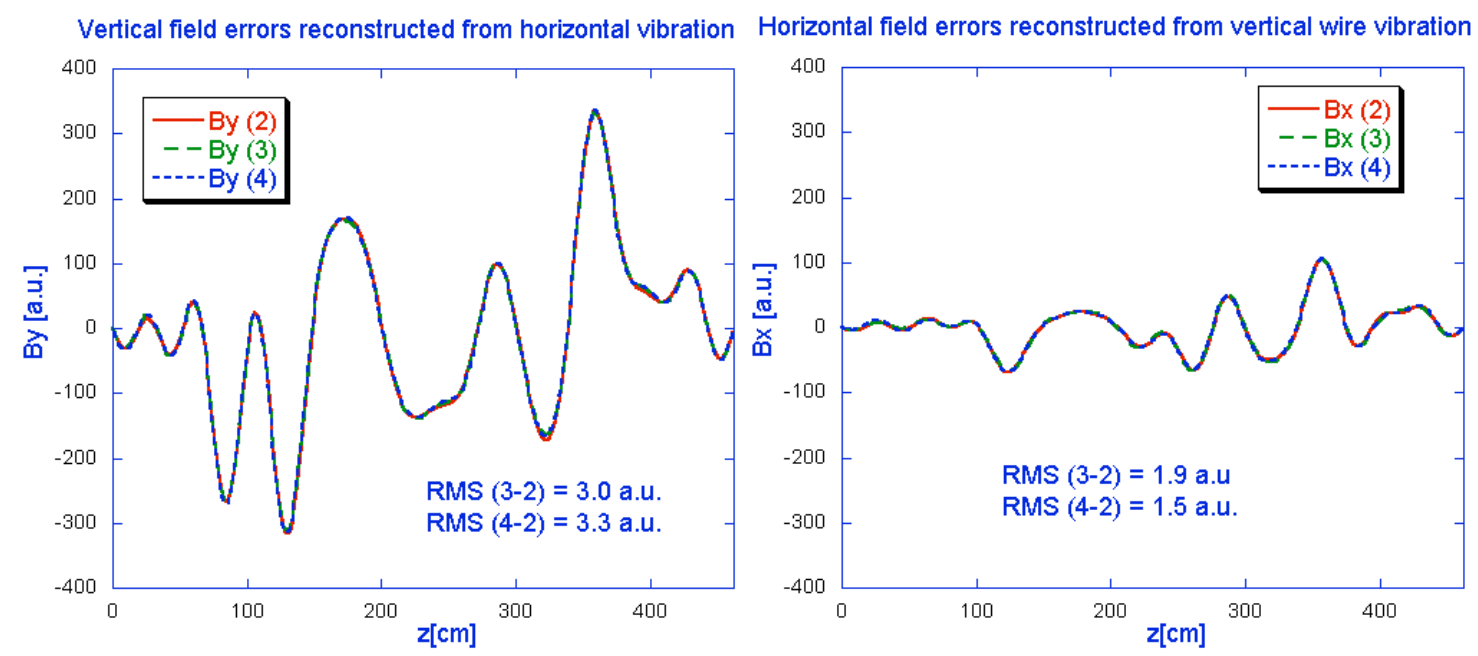

Figure 3:

Vertical (left) and horizontal (right) magnetic field reconstructed from the measured wire vibration modes. Three independent measurements are presented. The horizontal axis is the distance along the wire, vertical is a field component in arbitrary units.

According to this algorithm the reconstructed field as a function of distance along the wire, Fig. 3 , is the sum of the sinusoidal waves with amplitudes and phases presented in Fig. 2. The data indicate that repeatability is quite satisfactory. The RMS of the difference between independent measurements is approximately 3 arbitrary units; that is $\sim 1 \%$ of the signal maximum. Note, calibration described in the following section indicated that 3 a.u. corresponds to approximately 0.1 Gauss field. That is $\sim 0.7 \times 10^{-5}$ of the undulator peak field.

\section{Measurement of localized horizontal field variation.}

The next experiment was the measurement of a localized horizontal field. The field component was created by a standard shim used for vertical beam trajectory correction. The shim was placed at location $\mathrm{z}=231 \mathrm{~cm}$. Figure 4 depicts the difference in the vibrating wire harmonics (left) and in reconstructed fields (right) between measurements before and after shim placement. The harmonics structure corresponding to "delta-like" function and well defined peak in reconstructed field is clearly visible. A quadratic fit of the maximum, see insertion, gives the location of the peak at $\mathrm{z}=230.8 \mathrm{~cm}$, which is very close to the shim location $(231 \mathrm{~cm})$. 

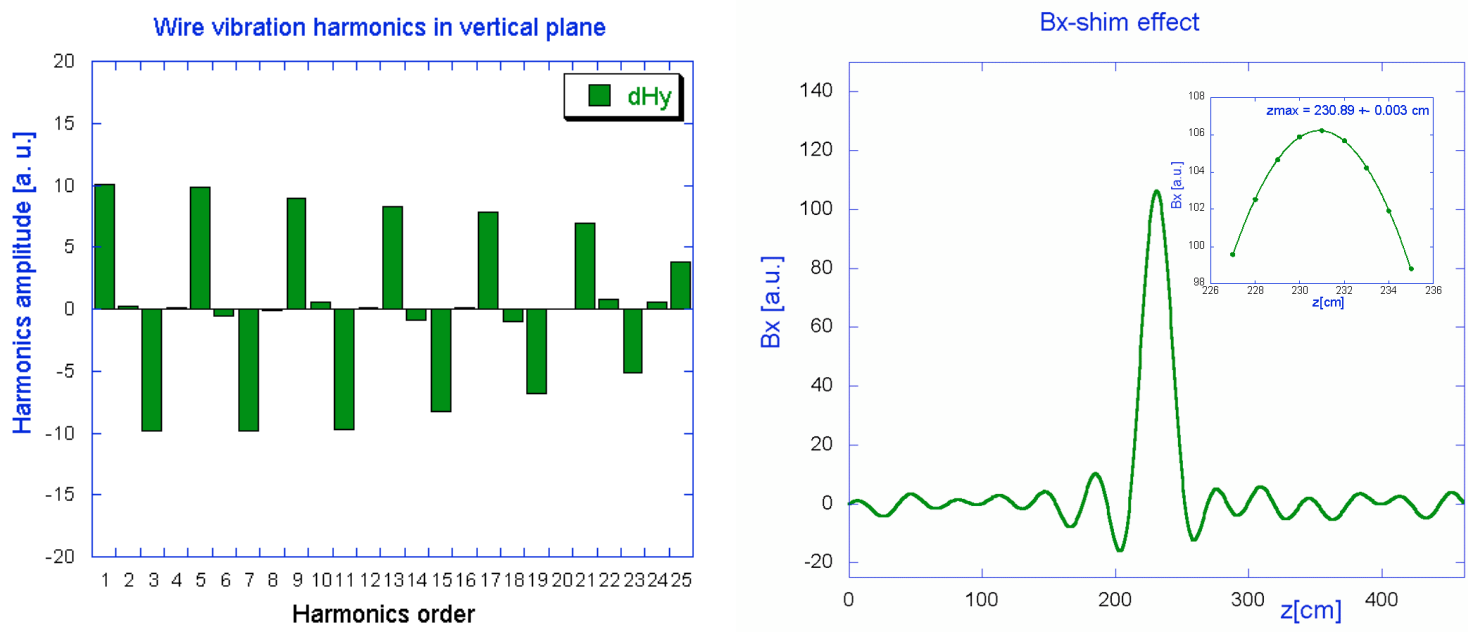

Figure 4:

Variation in the vertical vibrating harmonics (left) and in reconstructed field (right) caused by Bx-shim placed at $\mathrm{z}=231 \mathrm{~cm}$. Quadratic fit of the peak maximum, see insertion, gives the peak location $230.89 \mathrm{~cm}$.

The symmetric ripple seen around the peak is due to the limited number of the harmonics used for reconstruction. This ripple can be eliminated by applying an appropriate filter.

\section{Measurement of localized vertical field variation and signal calibration}

The effect of a single By-shim placed at $z=240 \mathrm{~cm}$ is shown on Fig.5. The plots present the difference in the wire vibration harmonics and reconstructed field measured before and after of the shim placement. A well-defined peak is present at $\mathrm{z}=241.1 \mathrm{~cm}$, which is very close to the actual shim position.
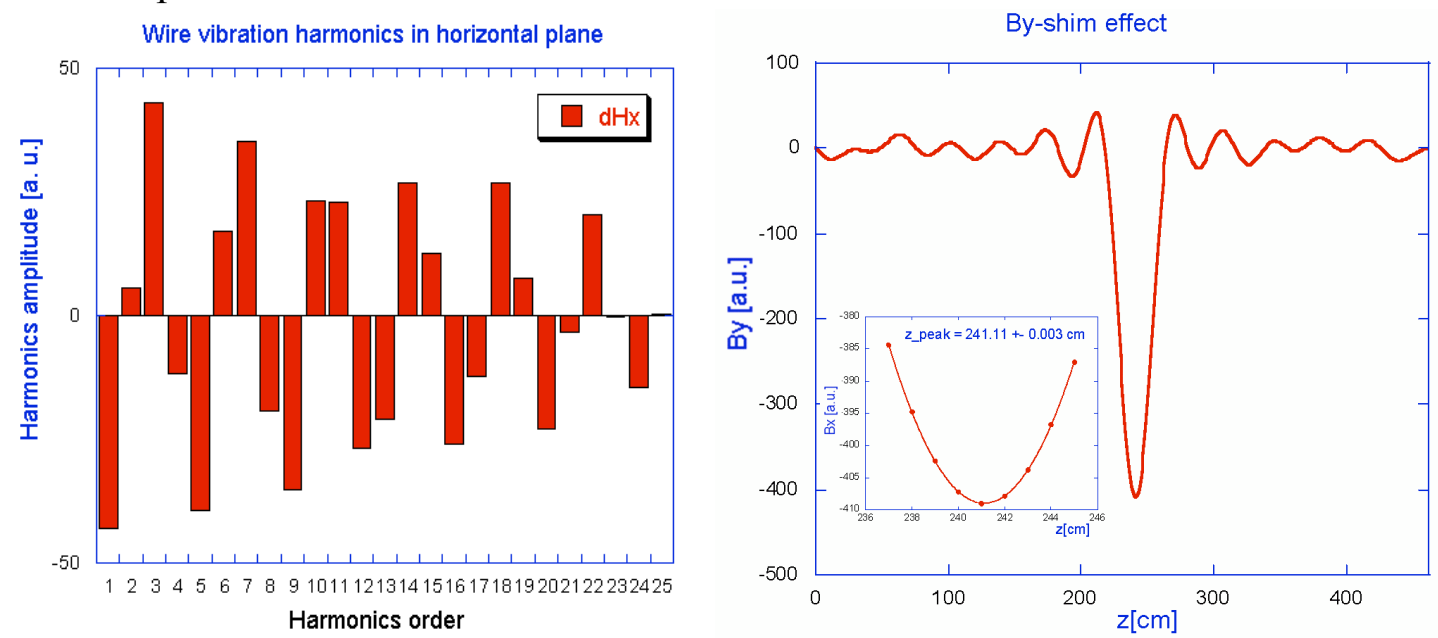

Figure 5:

Variation of the wire vibration harmonics (left) and reconstructed vertical field (right) caused by By-shim placed at $\mathrm{z}=241 \mathrm{~cm}$. Quadratic fit of the top of the peak gives the peak location $\mathrm{z}=241.1 \mathrm{~cm}$. The peak area (reconstructed field integral) is 10240 [a.u. $\mathrm{x} \mathrm{cm}$ ].

The peak area, i.e. integral of the reconstructed field over "z", is found to be 10240 [a.u. x cm]. From Hall probe measurement we know this type of shims generates local field distortion with 
$\sim 339 \mathrm{Gcm}$ integral. Comparing these numbers, one can find that 1a.u. of the reconstructed field corresponds to $3.32 \mathrm{e}-2$ Gauss.

\section{Space resolution test.}

In this test we compared field variations created by two shims placed on two neighboring poles $1.5 \mathrm{~cm}$ apart. Data on the left plot in Fig.6 show two reconstructed fields created by By-shims placed on pole \#122 and \#121. Note that the shim strength was significantly reduced in comparison with measurement presented in Fig.5.
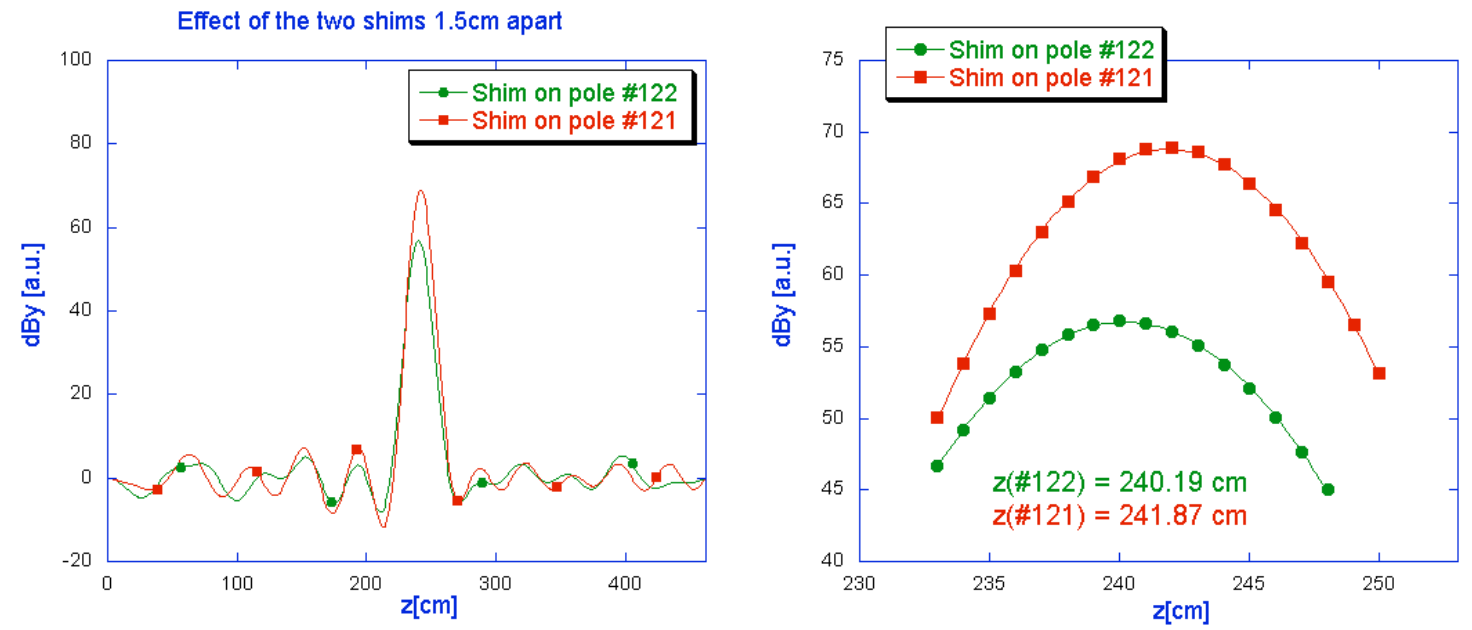

Figure 6:

Field created by shims placed $1.5 \mathrm{~cm}$ apart. On left plot is a reconstructed field profile along the wire. Quadratic fit, see plot on the right, gives the difference between peak locations $1.7 \mathrm{~cm}$. The expected distance is $1.5 \mathrm{~cm}$.

The measurement indicates $1.7 \mathrm{~cm}$ distance between peaks, which is $2 \mathrm{~mm}$ longer than the expected $1.5 \mathrm{~cm}$. In the second experiment we measured vertical field distortions created by two shims placed on poles separated by $6 \mathrm{~cm}$. Data are plotted in Fig. 7.
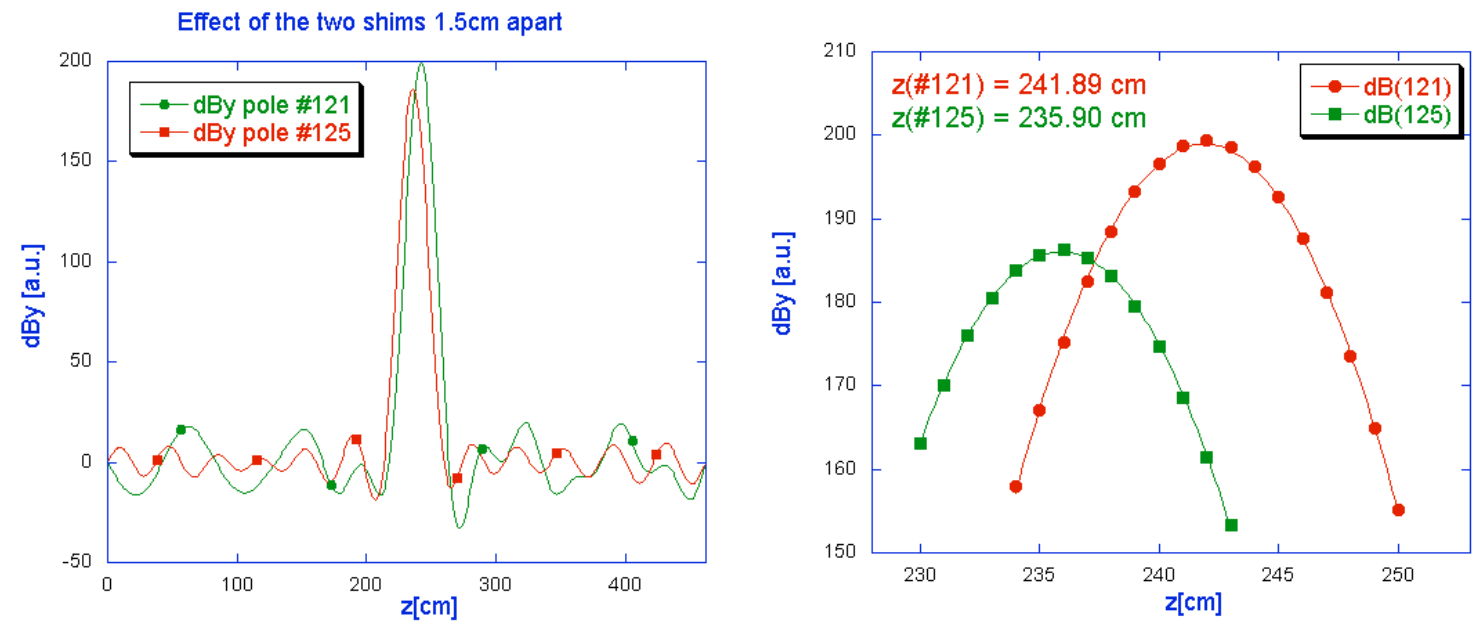

Figure 7:

Effect of two shims placed on poles \#121 and \#125 (6cm apart), see left plot. The distance between two peaks seen in reconstructed field, see right plot, is $6.01 \mathrm{~cm}$. The expected distance is $6.0 \mathrm{~cm}$. 
The distance between two peaks seen in measurement, $6.01 \mathrm{~cm}$, is very close to the expected $6 \mathrm{~cm}$.

Results of the measurement when two By-shims were placed on pole \#225 and pole \#42 are shown on Fig.8. In this case the distance between shims was $(225-42) \times 1.5 \mathrm{~cm}=274.5 \mathrm{~cm}$.

Two shims (pole \#42 and pole \#225) data

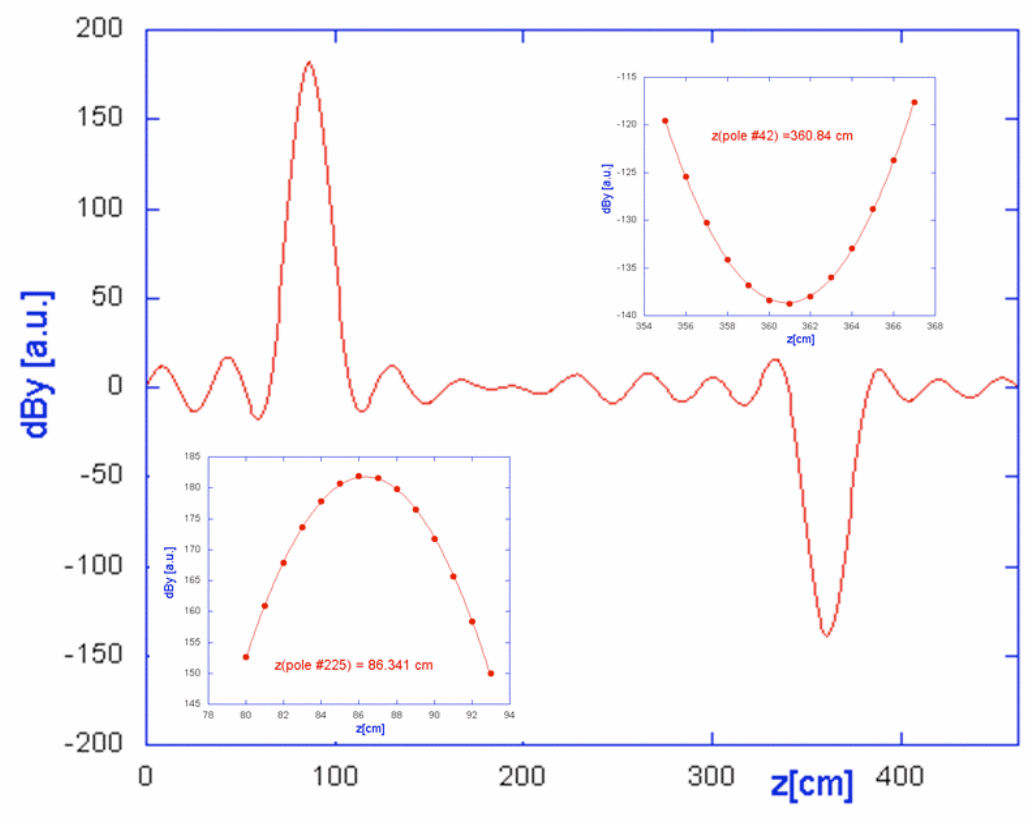

Figure 8:

Reconstructed field generated by two shims placed $274.5 \mathrm{~cm}$ apart on poles \#42 and \#225. Accurate fit, see insertions, gives $274.49 \mathrm{~cm}$ distance between peaks, while the expected is $274.5 \mathrm{~cm}$.

Accurate fit of the peak maximums shown on insertions gave $274.49 \mathrm{~cm}$ distance, while the expected is $274.5 \mathrm{~cm}$.

Comparing the measured and expected distances between locations of the field distortion we found the error RMS equals $0.9 \mathrm{~mm}$. This number gives the method's spatial resolution.

\section{Sensitivity test}

In a sensitivity test, we varied the strength of the By-shim placed on pole \#121 $(\mathrm{z} \sim 241 \mathrm{~cm})$ and measured amplitude of the 5-th mode of the wire vibration. Results are plotted on Fig. 8. The 5 th harmonic strength is plotted as a function of the shim's screw position, used to shunt magnetic field flux from the pole, as well as residuals from the linear fit. 


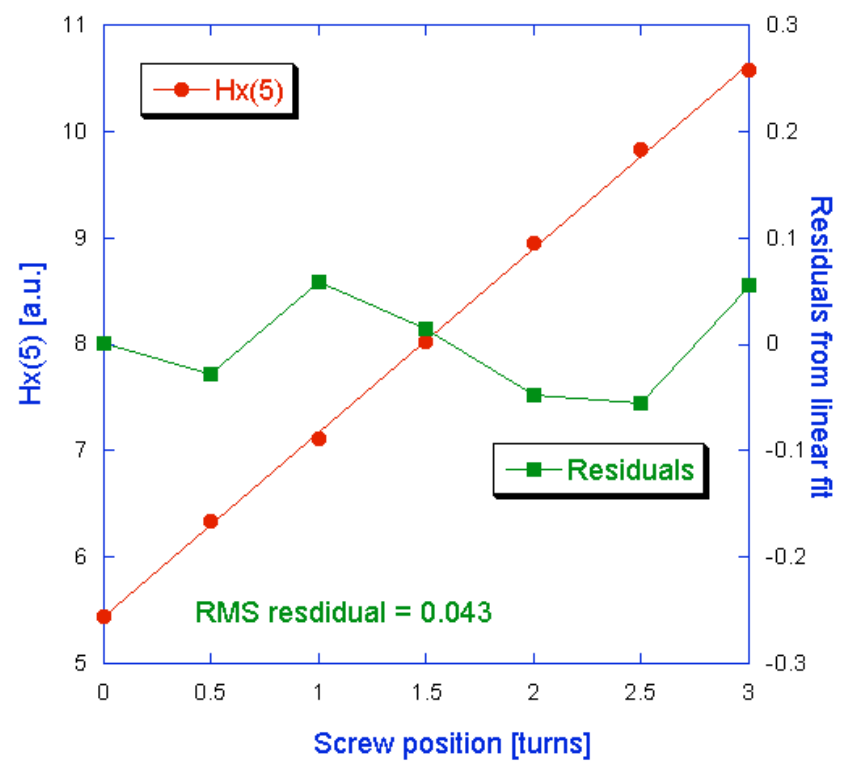

Figure 8:

The strength of the 5-th harmonic of wire vibration as a function of the strength of the By_shim placed on pole \#121 $(\mathrm{z}=241 \mathrm{~cm})$. The 0.043 a.u. of RMS of residuals corresponds to $0.37 \mathrm{Gcm}$ field integral.

We estimated the measurement errors, which determine the method sensitivity, by using the residuals from the linear fit. The RMS of the residuals was calculated as 0.043 a.u. According to calibration, $339 \mathrm{Gcm}$ of the field integral from the shim placed on pole \#122 results in 39.35 a.u. of the 5-th harmonic change, see left upper plot on Fig. 5. This implies that 1 a.u. of the harmonic's variation corresponds to $8.6 \mathrm{Gcm}$ of the field integral change. Consequently, 0.043 a.u. RMS of the harmonic strength measurement error translates to $0.37 \mathrm{Gcm}$ error in the field integral. The latter number can be considered as the method sensitivity. It should be added that this sensitivity exceeds the sensitivity which can be achieved with a Hall probe.

\section{Comparison with Hall probe measurement}

We compared magnetic field errors obtained with a Hall probe and with the Vibrating Wire technique. LCLS undulator (S/N 13) was put on the stand and characterized with Vibrating Wire. In characterization we used 25 harmonics of the wire vibration. The shortest halfwavelength equaled $18.5 \mathrm{~cm}$ or 6 (approximately) undulator periods. Plots in Fig. 9 depict the reconstructed field of the VW technique in comparison with the field measured with a Hall probe. The later was averaged over 6 undulator periods, i.e., over the shortest half-wavelength. Note that this averaging zeroed the ideal undulator field of $\sim 12,500 \mathrm{G}$ amplitude, and left the field errors with amplitude $\sim 1.7 \times 10^{-3}$ of the peak field. In the case of the vertical field (left plot) there are two regions at the undulator ends with bigger amplitudes. These regions represent end transitions where the undulator field is not periodic. For the Vibrating Wire field reconstruction we used the calibration obtained in the previous section. To give a sense of VW measurement repeatability on both plots (left and right) we depicted two independent sets of VW measurement data. At the given scale, they are undistinguishable. 

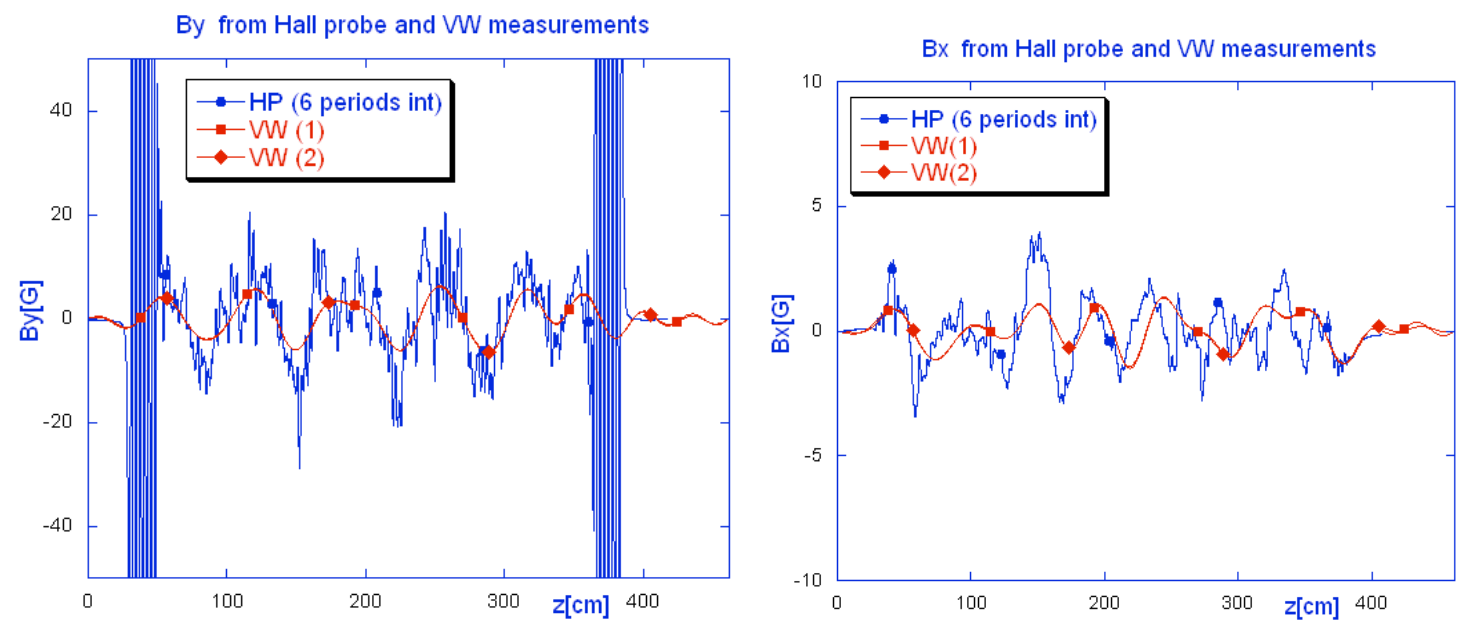

Figure 9:

Comparison between undulator field errors measured with Hall probe and Vibrating Wire. To obtain field errors from Hall Probe measurements, the field was averaged over 6 undulator periods.

Field error profiles obtained with the Hall probe and Vibrating Wire reveal similar structure and amplitudes. Both show approximately $\sim 15$ Gauss in the vertical field errors, and smaller, $\sim 3$ Gauss in the horizontal field errors.

Comparison between calculated 13.4 GeV LCLS beam trajectories based on measured fields by Hall probe and Vibrating Wire techniques is given in Fig.10. The calculated trajectories from the two techniques show good agreement.
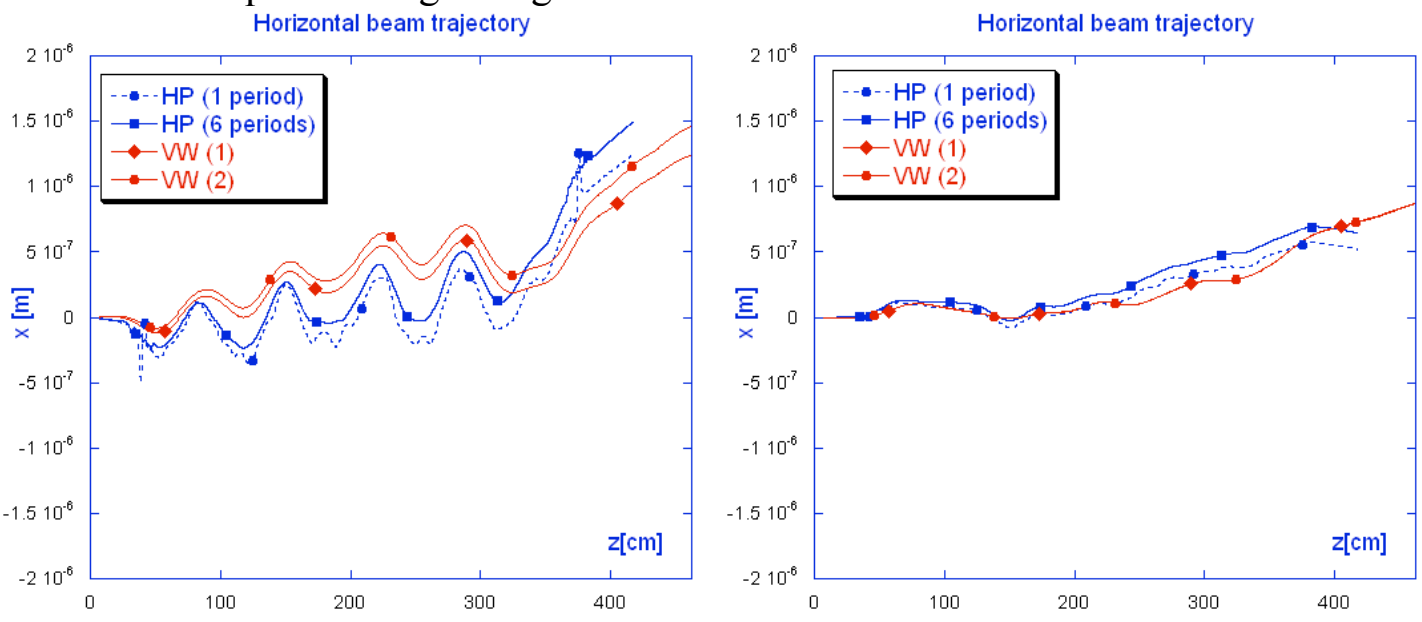

Figure 10:

Beam trajectories in horizontal (left) and vertical (right) planes calculated for Hall probe and Vibrating Wire measured field. Plots marked as "HP (1 period)" and "HP(6 periods)" represent trajectories calculated for Hall probe measured field averaged over one and 6 undulator periods. Plots labeled as "VW (1)" and "VW (2)" represent two independent VW measurements.

To explore the effect of the Hall probe field averaging, we calculated trajectories for the field averaged over 1 and 6 undulator periods, see plots marked as " HP (1 period)" and "HP(6 periods)". As one can see in Fig.10, the difference between them is not significant. This validates calculating beam trajectories using the undulator field which is averaged (or integrated) 
over multiple undulator periods. Note that the Vibrating Wire technique performs this integration automatically.

\section{Discussion and Conclusion.}

Using LCLS undulators as a test bench, we explored the potential of the Vibrating Wire technique for undulator field error measurement. The technique demonstrated:

a) $0.37 \mathrm{Gcm}$ sensitivity for the measurement of the magnetic field integrals;

b) $\sim 1 \mathrm{~mm}$ space resolution for locating the magnetic field distortion;

c) Satisfactory repeatability

Beam trajectories calculated based on Hall probe and Vibrating Wire field measurements are in good agreement.

The small size of the magnetic field sensitive element (stretched wire) and the setup simplicity of the Vibrating Wire field measurement technique enable the characterization of magnets with small apertures and limited access to the tested field region. In addition, because the VW method automatically integrates the field over the length of the standing wave, the signal is sensitive to the field errors, but not to the main field. This results in very high sensitivity, which is difficult to achieve in Hall probe measurements. Also, this technique is free from effects analogous to the planar Hall effect. The latter feature might be critical in the case of elliptical undulator tuning.

It should be noted that while the VW technique is more suitable for evaluation of the beam trajectory, it cannot be used for the measurement of undulator parameter " $\mathrm{K}$ ", the variation of which along the magnet causes optical phase shake and compromises undulator magnet performance. Thus, the VW technique cannot completely replace the Hall probe measurement technique, and should be considered as a supplement.

\section{Acknowledgment}

Authors would like to thank Animesh Jain for useful discussions and help in measurements. This work has been supported by NSF grant DMR 0225180 and in part by the DOE Contract DE-AC02-76SF00515 and was performed in support of the LCLS project at SLAC.

\section{References}

[1] A. Temnykh, Nucl, Instr. And Meth. 399 (1997) 185

[2] A. Temnykh, Magnetic survey of CESR final focus quadrupole magnets. Presentation on 13th International Magnetic Measurement Workshop, May 19-22, 2003, Stanford, California. URL: http://www.slac.stanford.edu/cgi-wrap/getdoc/slac-wp-029-ch27-Temnykh2.pdf

A. Temnykh, The Use of Vibrating Wire Technique for Precise Positioning of CESR Phase III Super- Conducting Quadrupoles at Room Temperature. In Proceedings of the 2001 Particle Accelerator Conference, Chicago, pp. 3469-3471. URL: http://accelconf.web.cern.ch/AccelConf/p01/PAPERS/RPPH100.PDF 
[3] A. Temnykh, Application of the Vibrating Wire Technique for Solenoid magnetic center finding. In proceedings of 14th International Magnetic Measurement Workshop 26-29

September 2005, Geneva, Switzerland

[4] A. Temnykh, Some Aspects of the Use of Vibrating Wire Technique for a Wiggler Magnetic Field Measurement, Preprint CBN 01-17, Cornell University 2001.

Alexander B. Temnykh and Kenneth D. Finkelstein, THE CHESS G-LINEWIGGLER TUNING. In Proceedings of the 2001 Particle Accelerator Conference, Chicago, pp. 2456-2458.

URL: http://accelconf.web.cern.ch/AccelConf/p01/PAPERS/WPPH004.PDF

A. Temnykh, VIBRATING WIRE AND FLIPPING COIL MAGNETIC MEASUREMENT OF A CESR-C 7-POLE WIGGLER MAGNET, In proceedings of the 2003 Particle Accelerator Conference, pp. 1026-1028. URL:

http://accelconf.web.cern.ch/AccelConf/p03/PAPERS/MPPG007.PDF

[5] Zachary Wolf, A Vibrating Wire System For Quadrupole Fiducialization, LCLS-TN-05-11, SLAC 2005. URL: http://www-ssrl.slac.stanford.edu/lcls/technotes/lcls-tn-05-11.pdf

[6] L. Tsai et al., MULTIPLE QUADRUPOLE MAGNETIC CENTER ALIGNMENT ON THE GIRDER, In proceedings of PAC07, Albuquerque, New Mexico, USA, pp. 395-397 URL: http://accelconf.web.cern.ch/AccelConf/p07/PAPERS/MOPAN100.PDF

[7] Animesh Jain et al., VIBRATING WIRE R\&D FOR ALIGNMENT OF MULTIPOLE MAGNETS IN NSLS-II, In proceedings of The 10th International Workshop on Accelerator Alignment, KEK, Tsukuba, 11-15 February 2008, URL:

http://www.slac.stanford.edu/econf/C0802113/papers/P019.pdf

Animesh Jain, Results from Vibrating Wire R\&D for Alignment of Multipoles in NSLS-II*, Presentation on 16th International Magnetic Measurement Workshop (IMMW16) Bad Zurzach, Switzerland, 26-29 October 2009, URL:

http://immw16.web.psi.ch/Presentations/2 18 IMMW16 Fiducialization.pdf 